\title{
A short story of $3 A B-O S$ cancer stem cells, a possible model for studying cancer stemness
}

\author{
Riccardo Di Fiore ${ }^{1}$, Rosa Drago-Ferrante ${ }^{1}$, Michela Marcatti ${ }^{1}$, Daniela Carlisi ${ }^{2}$, Giovanni Tesoriere ${ }^{3}$, Renza Vento ${ }^{1,3}$ \\ ${ }^{1}$ Laboratory of Biochemistry, Department of Biological, Chemical and Pharmaceutical Sciences and Technologies, University \\ of Palermo, Polyclinic, Palermo, Italy \\ ${ }^{2}$ Laboratory of Biochemistry, Department of Experimental Biomedicine and Clinical Neurosciences, University of Palermo, \\ Polyclinic, Palermo, Italy \\ ${ }^{3}$ Institute for Cancer Research and Molecular Medicine and Center of Biotechnology - College of Science and Biotechnology, \\ Temple University, Philadelphia (USA)
}

Correspondence: Renza Vento

E-mail: renza.vento@unipa.it

Received: April 02, 2014

Published online: October 10, 2014

\begin{abstract}
Cancer Stem Cells (CSCs) are thought to be the cause of cancer initiation, growth and development. Thus, a challenge in cancer research is their identification and eradication. In our laboratory, by chemical treatment of the human osteosarcoma (OS) MG63 cell line, we have isolated and characterized 3AB-OS cells, a human OS CSC line. 3AB-OS cells transdifferentiate in vitro into cells of the three derivatives germ layers and, when xenografted in athymic mice they are highly tumorigenic and recapitulate in vivo crucial features of human OS. They even express a reprogrammed energy metabolism, with a dependence on glycolytic metabolism more strong than parental MG63 cells. 3AB-OS cells have chromosomes showing a great number of abnormalities which are very similar to abnormalities found in both pediatric and adult osteosarcomas. In comparison with parental MG63 cells (where TP53 gene is hypermethylated, rearranged and in single copy), 3AB-OS cells have TP53 gene unmethylated, rearranged and in multiple copies. Moreover, the mutp53 (p53-R248W/P72R) is post-translationally stabilized, has nuclear localization and a gain of function. A great number of results obtained in our laboratories suggested that p53 mutation could be the "driver mutation" at the origin of the transformation of MG63 cells into 3AB-OS CSCs.
\end{abstract} cells

Keywords: Human osteosarcoma; cancer stem cells; mutant p53 gain of function; cancer cell dedifferentiation; $3 \mathrm{AB}-\mathrm{OS}$

Cancer Cell \& Microenvironment 2014; 1: e134 doi: 10.14800/ccm.134; ( 2014 by Riccardo Di Fiore, et al.

Cancer can develop in anyone with the risk of having a cancer increasing with age. Thus cancers are more numerous in adults, with $77 \%$ of them being diagnosed in persons older than 55 years of age ${ }^{[1]}$. Overall, death from cancer in the US accounts for about $20 \%$ of death ${ }^{[2]}$. The largest number of cancer patients live in low- and middle-income countries, where the medical resources and health systems are unable to coping with the disease. This is exacerbated by the evidence that the health burden of cancer grows in parallel with the population aging, in such a way that in 2030 more that 20 million new cancer cases are expected, accompanied by about 13 million of cancer deaths ${ }^{[3]}$. Terribly, the future may be much worse because of the lifestyles associated with urbanization and economic development (physical inactivity, smoking, environmental deterioration) in low- and middle-income countries. Thus, the most important challenge for cancer researchers is to reduce the enormous burden of cancer 
on public health.

Osteosarcoma (OS) is the most common bone cancer. Most osteosarcomas are treated with chemotherapy given before surgery (neoadjuvant chemotherapy) for about 10 weeks, followed by local tumor control (surgery and/or radiation) and, after that, again by chemotherapy (adjuvant chemotherapy) for up to a year. Currently,with the standard chemotherapy (cisplatin, doxorubicin and methotrexate) only $65-70 \%$ OS patients has a long-term survival disease-free without metastasis ${ }^{[4]}$, and in addition, for relapsed OS there is no second-line chemotherapy. The survival rates have essentially plateaued over the last several decades, particularly for patients with metastatic disease. Despite efforts to intensify therapy through dosing, timing or ablation, improvements have been limited. Thus efforts are currently focused on understanding the molecular and cellular mechanisms and signaling pathways involved in the pathogenesis and metastasis of OS in order to devise more rationale, targeted therapies aimed at improving long term patient survival.

It is known that solid tumors are organized hierarchically and that a distinct subpopulation of cells, termed cancer stem cells (CSCs), is at the root of their growth ${ }^{[5]}$, as they are responsible for tissue renewal, malignancy and resistance to therapies. However, still now the origin of CSCs is an outstanding issue, indeed it is not known whether CSCs arise from transformed stem cells or whether cancer cells are progressively transformed in CSCs. Anyway, it is certain that CSCs are present in the majority of tumors where they contribute to the initiation and development of the cancer [6] According to this knowledge, a fundamental problem in the research on OS is the identification of the CSCs within the cancer cell population with the aim of their eradication.

A human OS CSC line (3AB-OS) has been produced and isolated for the first time in our laboratory from the human osteosarcoma MG63 cell line by chemical treatment ${ }^{[7]}$. In this regard, we first showed that treating for a short time (24-72 h) MG63 cells with a competitive inhibitor of poly (ADP-ribose) polymerase, the 3-aminobenzamide $(3 \mathrm{AB})$, their growth rate resulted markedly reduced and this was accompanied by osteocyte differentiation ${ }^{[8]}$. We also observed that osteocyte differentiation was accompanied by reprogrammed gene expression, with down-regulation of genes required for proliferation and up-regulation of those implicated in osteoblastic differentiation ${ }^{[9]}$. Thereafter, studying the effects of the $3 \mathrm{AB}$ treatment for longer times (about 100 days) we observed the death of osteocyte cells with a simultaneous and progressive enrichment of a new, stable cell population, termed $3 \mathrm{AB}-\mathrm{OS}$. The characterization of $3 \mathrm{AB}-\mathrm{OS}$ cells demonstrated that they are a heterogeneous and stable cell population. Indeed, after serial passages (currently, more than 200) in the absence of $3 \mathrm{AB}$ this cell population retained all morphological and antigenic features. Importantly, 3AB-OS cells show unlimited proliferative potential, ABCG2-dependent phenotype with high drug efflux capacity, strong expression of stemness and antiapoptotic genes which are the most important features of CSCs ${ }^{[7]}$.

Multilineage differentiation potential is the important characteristic of CSCs, and there is now increasing evidence that microenvironments play critical roles in the behavior of stem cells, especially in deciding their differentiation directions ${ }^{[10]}$. Aimed at understanding the level of stemness of 3AB-OS CSCs, we showed that they are highly aggressive; placed in plates characterized by ultralow-attachment, they grow at a low density with a high sphere-formation efficiency ${ }^{[7]}$. They also penetrate Matrigel with an invasion ability which was 2.6-fold higher than parental MG63 cells. We even showed that $3 \mathrm{AB}-\mathrm{OS}$ cells transdifferentiate, in vitro, into cells of ectodermal, endodermal and mesodermal germ layers and that each of they showed specific morphological and functional properties ${ }^{[11]}$. When xenografted in athymic mice in the presence of matrigel, 3AB-OS cells were highly tumorigenic and induced the formation of a great number of blood vessels; they also showed multilineage commitment particularly favoring the mesenchymal lineage, with activation of myoblast differentiation and with the appearance of muscle fibers in the tumor mass. Overall, for the first time we provided a mouse model which recapitulate in vivo the key aspects of human osteosarcoma CSCs. Using this animal model, the efficacy in vivo of novel therapeutic treatments could be tested and predicted. Our results suggest that $3 \mathrm{AB}-\mathrm{OS}$ cells behavior might be regulated by matrigel interaction with subcutaneous microenvironment, with such interaction providing the adequate cues for transducing signals either oncogenic or differentiative ${ }^{[12]}$.

It is maintained that all cancers result from a process of Darwinian evolution developed among cell populations surrounded by various microenvironments provided by the tissues of a multicellular organism ${ }^{[13]}$. Similarly to the Darwinian evolution supporting the origins of species, cancer is assumed to originate by acquisition of genetic variation determined by random mutation in individual cells and by natural selection of the phenotypic diversity resultant by these mutations. Cells carrying deleterious mutations may be uprooted by natural selection, whereas cells carrying mutations conferring capability to proliferate and survive more efficiently than others may be favored. When casually a 
single cell acquires a number of favorable mutations, this will allow the cell to proliferate autonomously, invade and metastasize ${ }^{[13]}$. During the multistep development of human tumors, the cells acquire a number of biological capabilities (uninterrupted proliferative signaling, evasion of growth suppressors, cell death resistance, replicative immortality, angiogenesis induction, invasion and metastasis activation) which are recognized as cancer superpowers or hallmarks ${ }^{[14]}$. The acquisition of these hallmarks is enabled by genomic instability, which in turn generates the genetic diversity that accelerates their acquisition. Recently, an emerging hallmark has been identified in the "reprogramming energy metabolism", a series of adjustments of energy metabolism aimed at fueling cell growth and division. It may not be surprising that growing tumors, which have potent increases in cell proliferation accompanied by environmental fluctuations in nutrient and oxygen availability, display significant changes in pathways of energy metabolism and nutrient uptake. The first observation that tumor cells, to produce ATP from glucose, prefer to use glycolysis instead than oxidative phosphorylation, was made by Otto Warburg which suggested that defects in mitochondrial function, could be at the root of cancer ${ }^{[15]}$. Glycolytic fueling has been shown to be associated with mutations in tumor suppressors genes (e.g., TP53), whose alterations in tumor cells are selected as they avoids cytostatic controls, and attenuates apoptosis, thus conferring the hallmark capabilities for cell proliferation [16]. Recently, bioenergetics studies have revealed that human ESCs depend on glycolysis for ATP production [17] Consistently, mitochondria are less complex and fewer in number in human ESCs than in their differentiated progeny, and it has been shown that a glycolytic engagement is a crucial step in the conversion of terminally differentiated cells into iPSCs ${ }^{[18]}$. However, still now it is not known whether metabolic shifts precede pluripotency acquisition or stem cell state promote changes in metabolism. It has been suggested that during reprogramming a positive feedback loop is initiated, in which transcription factors first elicit a metabolic shift that is then necessary to induce additional endogenous pluripotency factors to complete the reprogramming into a stem cell state ${ }^{[19]}$. Overall, these results suggest that tumor bioenergetics and CSCs might be related to each other. To characterize the energetic properties of 3AB-OS CSCs, we analyzed their bioenergetics features comparatively with that of the parental more differentiate MG63 OS cells. Our results demonstrated that 3AB-OS cells depend on glycolytic metabolism more strongly than MG63 cells. Moreover, 3AB-OS cells show lower levels of mitochondrial respiration, are more sensitivity to glucose depletion or glycolytic inhibitors and show a reduced sensitivity to inhibitors of oxidative phosphorylation. In addition, with respect to MG63 cells, $3 \mathrm{AB}-\mathrm{OS}$ have fragmented mitochondria which rapidly networked in the presence of glucose-rich medium, whereas, growing in low glucose they entirely lose these structures. Overall, the findings suggested that the energy metabolism of 3AB-OS CSC strongly resemble that of normal stem cells and cancer cells which are characterized by a glycolytic anaerobic metabolism ${ }^{[20]}$.

Cancer cells have multiple genetic/epigenetic changes responsible for chromosomal instability and tumor progression ${ }^{[21]}$. These mechanisms involve aberrations with loss or gain of genes function that mostly impinge on tumor suppressors. In our laboratories we have shown that $3 \mathrm{AB}-\mathrm{OS}$ cells have large chromosomal complexity and molecular abnormalities which are present in the most aggressive human cancers. 3AB-OS cells have a hyper triploid karyotype and chromosome number ranging from 71 to 82 , whereasMG63 cells have a hypotriploid karyotype and chromosome number ranging from 61 to 66. Moreover, 3AB-OS cells show monosomies, trisomies and nullisomies, have 32 unidentifiable marker chromosomes, and exhibit -with respect to parental MG63 cells- 49 copy number variations (gains/losses) affecting almost all the chromosomes ${ }^{[22]}$. Intriguingly, the abnormalities evidenced in 3AB-OS cells are very similar to those described in a large number of pediatric and adult osteosarcomas, where karyotypes ranging from haploid to near hexaploid have been shown ${ }^{[23-25]}$. Moreover, $3 \mathrm{AB}-\mathrm{OS}$ cells showed losses/gains in agreement with those seen in osteosarcoma patients ${ }^{[23-25]}$. Comparing $3 \mathrm{AB}-\mathrm{OS}$ cells to MG63 cells we reported the gene expression profile of $3 \mathrm{AB}-\mathrm{OS}$ cells. In addition, employing KEGG and BioCarta analysis, we selected 196 genes which resulted up-/down-regulated and which appeared to be involved in regulatory pathways of tumorigenesis and stemness (ECM-receptor interaction and cell communication; cell adhesion molecules; ABC transporters in general; Notch and Hedgehog signaling; MAPK signaling; cell cycle regulators; apoptosis) most of which have been reported in osteosarcoma patients ${ }^{[26]}$. In addition, by human MicroRNA array analyses we identified 189 differentially expressed miRNAs and the analysis of mRNA/miRNA expression profiles appeared to be very similar to those reported in human OS patients ${ }^{[27,28]}$. Furthermore, to define a surface protein signature specific for 3AB-OS CSCs and MG63 cells, we have used cytometric analysis with an antibody-array comprising 245 membrane proteins. We also selected MAPK/ERK1/2 as a potential protein kinase-pathway correlated with pathways that characterize tumorigenesis and stemness of $3 \mathrm{AB}-\mathrm{OS}$ cells ${ }^{[29]}$.

More than half of all cancers exhibit mutation at p53 
locus on the short arm of chromosome I7 at I7p13 ${ }^{[30]}$. As a consequence, the most important tumor suppressor (p53) results altered in its functions as it may result either mutated or lost. Wild-type human p53 transcriptionally targets a large number of genes which mediates cell cycle arrest, DNA repair, senescence, apoptosis, and various metabolic processes. Thus, the loss of p53 function has been usually associated with an impairment of these functions ${ }^{[31]}$. TP53 missense mutations represent the majority of cancer-associated mutations which lead to the expression of a full-length altered p53 protein which, in contrast to wild-type p53, has a prolonged half-life. These missense mutations even determine oncogenic or gain-of-function activity ${ }^{[32,33]}$. Moreover, the presence of mutant p53 with gain of functions introduces new components which activate cancer progression and aggressiveness ${ }^{[34,35]}$. Most recently ${ }^{[36]}$, our studies on TP53 gene status/role performed in the highly aggressive 3AB-OS CSC line, have shown its involvement in promoting proliferation, invasiveness, resistance to apoptosis and stemness. In particular, we have shown that, in comparison with parental MG63 cells (where TP53 gene is hyper methylated, rearranged and in single copy), 3AB-OS cells have TP53 gene unmethylated, rearranged and in multiple copies. Moreover, mutp53 (p53-R248W/P72R) is post-translationally stabilized and with nuclear localization. Interestingly, p53-R248W/P72R-knockdown by short-interfering RNA, reduced the growth and replication rate of $3 \mathrm{AB}-\mathrm{OS}$ cells, markedly increasing cell cycle inhibitor levels and sensitizing 3AB-OS cells to TRAIL-induced apoptosis; in addition, the knockdown strongly decreased the levels of stemness and invasiveness genes; finally, the ectopic expression of p53-R248W/P72R in parental MG63 cells promoted cancer stem-like features (high proliferation rate, sphere formation, clonogenic growth, high migration and invasive ability) and strongly increased the levels of stemness proteins (CD133, ABCG2, Nanog, OCT3/4, Nucleostemin, Sox2). Overall, the findings suggested that p53 mutation could be the "driver mutation" at the origin of the dedifferentiation of MG63 cells into 3AB-OS CSCs.

Currently, we are working at the following purposes: 1) we have previously described in 3AB-OS CSCs a predictive network for let-7/98 and miR-29a, b, c, (two deeply down regulated miRNA family) and MSTN, CCND2, Lin28B, MEST, HMGA2, GHR (their anticorrelated and highly up regulated mRNAs). These miRNAs/mRNAs may represent new biomarkers for OS and permit the identification of new potential therapeutic targets ${ }^{[22]}$. Now, we have singularly over expressed these miRNAs in 3AB-OS cells and we are aimed at studying their effects on $3 \mathrm{AB}-\mathrm{OS}$ stemness and behaviour. To do this, we will subcutaneously xenograft athymic nude mice, separately, with each of the 3AB-OS clones overexpressing these miRNAs, in comparison with mice xenografted with parental $3 \mathrm{AB}-\mathrm{OS}$ cells; 2) because we think that p53 mutation could be the "driver mutation" at the root of the dedifferentiation of MG63 cells into $3 \mathrm{AB}-\mathrm{OS}$ CSCs ${ }^{[36]}$, we are producing, by stable RNAi system, a $3 \mathrm{AB}-\mathrm{OS}$ cell line that does not express mutant p53. We aim to evaluate whether the $3 \mathrm{AB}-\mathrm{OS}$ cells lacking the mutant p53, undergo morphological, molecular and fuctional changes; 3) it is easily conceivable that the multipotent phenotype of CSCs can simultaneously detect and integrate multiple signals from their microenviroment and convert them to a coherent environmental signal to regulate the downstream gene expression and stem cell fate ${ }^{[10]}$. Given the pluripotent ability evidenced in vitro by $3 \mathrm{AB}-\mathrm{OS}$ CSCs, we will inoculate these cells in various heterotopic sites in athymic nude mice, aimed at evaluating whether the microenvironment is critical for CSCs in deciding differentiation directions.

\section{Acknowledgments}

This work was partially funded by European Regional Development Fund, European Territorial Cooperation 2007-2013, CCI 2007 CB 163 PO 037, OP Italia-Malta 2007-2013-ImaGenX; Italian Ministry of Education, University and Research (MIUR) ex-60\%, 2013; MIUR-PRIN, contract number 2008P8BLNF (2008). Daniela Carlisi is a recipient of a fellowship granted by MIUR (contract number 82/01/23/2014). Riccardo Di Fiore and Rosa Drago-Ferrante were recipients of fellowships granted by the European Regional Development Fund, European Territorial Cooperation 2007-2013, CCI 2007 CB 163 PO 037, OP Italia-Malta 2007-2013.

\section{References}

1. Global Cancer Facts \& Figures." 2011. American Cancer Society [http://www.cancer.org/research/cancerfactsfigures/ cancerfactsfigures/cancer-facts-figures-2011].

2. Siegel R, Naishadham D, Jemal A. Cancer statistics, 2012.CA Cancer J Clin 2012; 62:10-29.

3. Introduction World Health Organization (WHO): Cancer prevention and control; report to the secretariat by the 58th World Health Assembly. [http://www.who.int/mediacentre/ news/releases/2005/pr_wha05/en/index.html. Accessed January 23, 2012].

4. Bispo Júnior RZ, Camargo OP. Prognostic factors in the survival of patients diagnosed with primary non-metastatic osteosarcoma with a poor response to neoadjuvant chemotherapy. Clinics (Sao Paulo) 2009; 64:1177-1186.

5. Clevers H. The cancer stem cell: Premises, promises and challenges. Nat Med 2011; 17:313-319. 
6. Giordano A, Fucito A, Romano G, Marino IR. Carcinogenesis and environment: the cancer stem cell hypothesis and implications for the development of novel therapeutics and diagnostics. Front Biosci 2007; 12:3475-3482.

7. Di Fiore R, Santulli A, Ferrante RD, Giuliano M, De Blasio A, Messina $\mathrm{C}$, et al. Identification and expansion of human osteosarcoma-cancer-stem cells by long-term 3-aminobenzamide treatment. J Cell Physiol 2009; 219:301-313.

8. De Blasio A, Musmeci MT, Giuliano M, Lauricella M, Emanuele S, D'Anneo A, et al. The effect of 3-aminobenzamide, inhibitor of poly (ADP-ribose) polymerase, on human osteosarcoma cells. Int J Oncol 2003; 23:1521-1528.

9. De Blasio A, Messina C, Santulli A, Mangano V, Di Leonardo E, D'Anneo A, et al. Differentiative pathway activated by 3-aminobenzamide, an inhibitor of PARP, in human osteosarcoma MG63 cells. FEBS Lett 2005; 579:615-620.

10. Sun Y, Chen CS, Fu J. Forcing stem cells to behave: a biophysical perspective of the cellular microenvironment. Annu Rev Biophys 2012; 41:519-542.

11. Di Fiore R, Drago-Ferrante R, D'Anneo A, De Blasio A, Santulli A, Messina C, et al. Differentiation of human osteosarcoma $3 \mathrm{AB}-\mathrm{OS}$ stem-like cells in derivatives of the three primary germ layers as an useful in vitro model to develop several purposes. Stem Cell Discovery 2013; 3:188-201.

12. Di Fiore R, Guercio A, Puleio R, Di Marco P, Drago-Ferrante $\mathrm{R}$, D'Anneo A, et al. Modeling human osteosarcoma in mice through 3AB-OS cancer stem cell xenografts. J Cell Biochem 2012; 113:3380-3392.

13. Little MP. Cancer models, genomic instability and somatic cellular Darwinian evolution. Biol Direct 2010; 5:19.

14. Hanahan D, Weinberg RA. Hallmarks of cancer: the nextgeneration. Cell 2011; 144:646-674.

15. Warburg O. On the origin of cancer cells. Science 1956; 123:309-314.

16. Jones RG, Thompson CB. Tumor suppressors and cell metabolism: a recipe for cancer growth. Genes Dev 2009; 23:537-548

17. Zhou W, Choi M, Margineantu D, Margaretha L, Hesson J, Cavanaugh $\mathrm{C}$, et al. HIF1a induced switch from bivalent to exclusively glycolytic metabolism during ESC-toEpiSC/hESC transition. EMBO J 2012; 31:2103-2116.

18. Varum S, Rodrigues AS, Moura MB, Momcilovic O, Easley CA 4th, Ramalho-Santos J, et al. Energy metabolism in human pluripotent stem cells and their differentiated counterparts. PLoS One 2011; 6:e20914.

19. Shakya A, Cooksey R, Cox JE, Wang V, McClain DA, Tantin D. Oct1 loss of function induces a coordinate metabolic shift that opposes tumorigenicity. Nat. Cell Biol 2009; 11:320-327.

20. Palorini R, Votta G, Balestrieri C, Monestiroli A, Olivieri S, Vento R, et al. Energy metabolism characterization of a novel cancer stem cell-like line 3AB-OS. J Cell Biochem 2014; 115:368-379.

21. Lagasse E. Cancer stem cells with genetic instability: the best vehicle with the best engine for cancer. Gene Ther 2008; 15:136-142.

22. Di Fiore R, Fanale D, Drago-Ferrante R, Chiaradonna F,
Giuliano M, De Blasio A, et al. Genetic and molecular characterization of the human osteosarcoma $3 \mathrm{AB}-\mathrm{OS}$ cancer stem cell line: A possible model for studying osteosarcoma origin and stemness. J Cell Physiol 2013; 228:1189-1201.

23. Bridge JA, NelsonM, McComb E, McGuire MH, RosenthalH, VergaraG, et al. Cytogenetic findings in 73 osteosarcoma specimens and a review of the literature. Cancer Genet Cytogenet 1997; 95:74-87.

24. Batanian JR, Cavalli LR, Aldosari NM, Ma E, Sotelo-Avila C, Ramos MB, et al. Evaluation of paediatric osteosarcomas by classic cytogenetic and CGH analyses. Mol Pathol 2002; 55:389-393.

25. Niini T, Lahti L, Michelacci F, Ninomiya S, Hattinger CM, Guled M, et al. Array comparative genomic hybridization reveals frequent alterations of G1/S checkpoint genes in undifferentiated pleomorphic sarcoma of bone. Genes Chromosomes Cancer 2011;50:291-306.

26. Cleton-Jansen AM, Anninga JK, Briaire-de Bruijn IH, Romeo S, Oosting J, Egeler RM, et al. Profiling of high-grade central osteosarcoma and its putative progenitor cells identifies tumourigenic pathways. Br J Cancer 2009; 101:1909-1918.

27. Maire G, Martin JW, YoshimotoM, Chilton-MacNeill S, Zielenska M, Squire JA. Analysis of miRNA-gene expression-genomic profiles reveals complex mechanisms of microRNA deregulation in osteosarcoma. Cancer Genet 2011; 204:138-146.

28. Jones KB, Salah Z, Del Mare S, Galasso M, Gaudio E, Nuovo GJ, et al. miRNA signatures associate with pathogenesis and progression of osteosarcoma. Cancer Res 2012; 72:1865-1877.

29. Gemei M, Corbo C, D'Alessio F, Di Noto R, Vento R, Del Vecchio L. Surface proteomic analysis of differentiated versus stem-like osteosarcoma human cells. Proteomics 2013; 13:3293-3297.

30. Vogelstein B, Lane D, Levine AJ. Surfing the p53 network. Nature 2000; 408:307-310.

31. Vousden KH, Lu X. Live or let die: The cell's response to p53. Nat Rev Cancer 2002; 2: 594-604.

32. Strano S, Dell'Orso S, Di Agostino S, Fontemaggi G, Sacchi A, Blandino G. Mutant p53: An oncogenic transcription factor. Oncogene 2007; 26:2212-2219.

33. Oren M, Rotter V. Mutant p53 gain-of-function in cancer. Cold Spring Harb Perspect Biol 2010; 2:a001107.

34. Sarig R, Rivlin N, Brosh R, Bornstein C, Kamer I, Ezra O, et al. Mutantp53facilitatessomatic cell reprogramming and augments the malignant potential of reprogrammed cells. J Exp Med 2010; 207:2127-2140.

35. Muller PA, Vousden KH, Norman JC. p53 and its mutants in tumor cell migration and invasion. J Cell Biol 2011, 192:209-218.

36. Di Fiore R, Marcatti M, Drago-Ferrante R, D'Anneo A, Giuliano M, Carlisi D, et al. Mutant p53 gain of function can be at the root of dedifferentiation of human osteosarcoma MG63 cells into 3AB-OS cancer stem cells. Bone 2014; 60:198-212.

To cite this article: Riccardo Di Fiore, et al. A short story of $3 \mathrm{AB}-\mathrm{OS}$ cancer stem cells, a possible model for studying cancer stemness. Can Cell Microenviron 2014; 1: e134. doi: 10.14800/ccm.134. 